\title{
Somatic drivers of B-ALL in a model of ETV6-RUNX1; Pax5 $^{+/-}$leukemia
}

Louise van der Weyden ${ }^{1 \dagger}$, George Giotopoulos ${ }^{2 \dagger}$, Kim Wong ${ }^{1}$, Alistair G. Rust ${ }^{1}$, Carla Daniela Robles-Espinoza', Hikari Osaki ${ }^{2}$, Brian J. Huntly ${ }^{2}$ and David J. Adams ${ }^{1,3^{*}}$

\begin{abstract}
Background: B-cell precursor acute lymphoblastic leukemia (B-ALL) is amongst the leading causes of childhood cancer-related mortality. Its most common chromosomal aberration is the ETV6-RUNX1 fusion gene, with 25\% of ETV6-RUNX1 patients also carrying PAX5 alterations.

Methods: We have recreated this mutation background by inter-crossing Etv6-RUNX1 (Etv6 ${ }^{R U N X 1-S B}$ ) and Pax $5^{+/-}$mice and performed an in vivo analysis to find driver genes using Sleeping Beauty transposon-mediated mutagenesis and also exome sequencing.

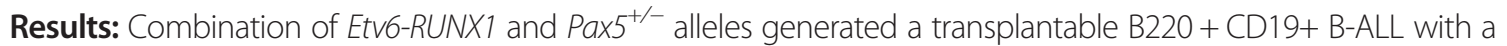
significant disease incidence. RNA-seq analysis showed a gene expression pattern consistent with arrest at the pre-B stage. Analysis of the transposon common insertion sites identified genes involved in B-cell development (Zfp423) and the JAK/STAT signaling pathway (Jak1, Stat5 and II2rb), while exome sequencing revealed somatic hotspot mutations in Jak 1 and Jak3 at residues analogous to those mutated in human leukemias, and also mutation of Trp53.
\end{abstract}

Conclusions: Powerful synergies exists in our model suggesting STAT pathway activation and mutation of Trp53 are potent drivers of B-ALL in the context of Etv6-RUNX1;Pax $5^{+/-}$.

Keywords: ETV6-RUNX1, Pax5, JAK/STAT, Trp53, Leukemia, B-cell precursor, Insertional mutagenesis

\section{Background}

B-cell precursor acute lymphoblastic leukemia (B-ALL) is the most common childhood tumor [1]. The most common chromosomal rearrangement in B-ALL is the $\mathrm{t}(12 ; 21)(\mathrm{p} 13 ; \mathrm{q} 22)$ translocation generating the ETV6RUNX1 fusion gene [2]. This fusion is necessary but insufficient for the development of B-ALL, as monozygotic twin studies, and the detection of the ETV6-RUNX1 fusion in fetal blood spots from patients who do not go on to develop B-ALL have shown [3, 4].

$P A X 5$, a guardian of B-cell identity and function, is somatically mutated in $\sim 40 \%$ of cases of childhood B-ALL [5]. Moreover, the most common recurrent focal deletion region in ETV6-RUNX1+ tumors involves PAX5 (9p13.2; 25 \%)

\footnotetext{
* Correspondence: da1@sanger.ac.uk

${ }^{\dagger}$ Equal contributors

'Wellcome Trust Sanger Institute, Wellcome Trust Genome Campus, Hinxton, Cambridge CB10 1HH, UK

${ }^{3}$ Experimental Cancer Genetics, Wellcome Trust Sanger Institute, Wellcome

Trust Genome Campus, Hinxton, Cambridge CB10 1HH, UK

Full list of author information is available at the end of the article
}

and these deletions are thought to be early events in leukemogenesis [6]. Previously, we generated a knock-in mouse model of ETV6-RUNX1 ALL, in which expression of the fusion gene is driven from the endogenous Etv6 promoter, and is linked to expression of the Sleeping Beauty (SB) transposase allowing the identification of transposon gene mutations that co-operate with Etv6RUNX1 in leukemia development [7]. Given that PAX5 heterozygosity is a frequent event in ETV6-RUNX1 patients [5], we bred these mice onto a background of Pax5 heterozygosity and performed a $S B$ transposon-mediated mutagenesis screen to explore the profile of co-operating drivers. We coupled this approach with targeted exome sequencing of tumors to find additional mutations, and in particular hotspot mutation events.

\section{Methods}

\section{Mouse strains}

Generation and genotyping of Etv6-RUNX1, T2Onc [7] and Pax5 [8] mice has been described previously. For secondary

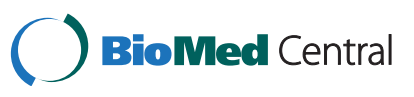


bone marrow transplants of tumors, $6-12$ week old SCID mice were inoculated with $3.5-5 \times 10^{5}$ bone marrow or spleen cells by tail vein injection. Animal studies were approved by the Home Office UK. Flow cytometric analysis of $\mathrm{CD}$ antigen expression was performed on single-cell suspensions from spleen or bone marrow as described previously [7].

\section{Identification and analysis of genes affected by SB mutagenesis}

Isolation of the transposon insertion sites and Gaussian kernel convolution statistical methods to identify common insertion sites (CISs) have been described previously [7]. Whole transcriptome sequencing (RNA-seq) was performed on splenic RNA using the mRNA Seq Sample Prep Kit (Illumina, San Diego, CA) to create libraries that were sequenced on the Illumina platform. HTSeq-counts (HTSeq framework; v0.54p5) were used as input to edgeR (v3.4.2). Genes with significant differential expression were defined based on an FDR of $5 \%$. Pathway and gene set enrichment analysis (GSEA) was performed using Ingenuity Pathway Analysis and GSEA (v2.0.14), respectively.

\section{Exome sequencing and bait design}

Spleen ('tumor') and tail ('normal') genomic DNA were extracted using the Gentra Puregene Cell Kit (Qiagen). Exon-coding sequences of genes previously found to be involved in cancer were captured using custom-designed baits (Additional file 1) and sequenced on an Illumina platform. For each tumor-normal pair, MuTect (v1.14) was used to identify somatic SNVs, which were annotated using the Variant Effect Predictor tool (Ensembl v74). The Jak1, Jak3 and Trp53 mutations were validated by capillary sequencing.

\section{Results and discussion}

To perform the $S B$ transposon-mediated mutagenesis screen we intercrossed $\operatorname{Pax5}\left(\operatorname{Pax}^{+/-}\right)$mice with transposon-carrying T2Onc mice and the resulting offspring were intercrossed with transposase-carrying Etv6RUNX1 (Etv6 $\left.{ }^{+/ R U N X 1-S B}\right)$ mice (Methods). The resulting genotypes in which transposition would occur were Etv6 ${ }_{+/ R U N X 1-S B}, \mathrm{~T}_{2} \mathrm{Onc}^{+/ T g}, \mathrm{Pax}^{+/-}$(hereafter referred to as ER, Onc, Pax) and Etv6 ${ }^{+/ R U N X 1-S B}, T 2 O n c^{+/ T g}$ mice (hereafter referred to as $E R, O n c)$. Importantly we found that $E R$, Onc, Pax mice showed a significant increase in the proportion of B-cell precursor (BCP)-ALL cases when compared to ER, Onc mice wildtype for Pax5 (41/159 (26\%) versus $1 / 37$ (3\%); p < 0.005 using a 2-tailed Fisher's exact test), with 27/41 (66 \%) of these cases being B220+ CD19+ (Fig. 1). Additional immunophenotyping of these $\mathrm{B} 220+\mathrm{CD} 19+$ cells from $E R$, Onc, Pax mice confirmed their ontogenic arrest at the pre-B stage (consistent with
Hardy fraction C'/D and ETV6-RUNX1+ patient leukemic cells; Fig. 1d). Importantly, the leukemias with an almost pure population of $\mathrm{B} 220+\mathrm{CD} 19+$ cells were also transplantable in SCID mice (with recipients developing B-ALL within 11-55 days; Fig. 1e). RNA-seq analysis performed on 20 B-ALL cases and 6 age-matched control cases (ER, Onc, Pax mice that never developed disease) revealed that 14/34 (41\%) differentially expressed genes were components of canonical B cell development pathways $\left(\mathrm{p}=1.26 \times 10^{-6}\right.$; Ingenuity Pathway Analysis), while GSEA revealed a significant enrichment for genes up-regulated in early B-cell development, specifically the pre-B stage (Additional file 2: Figure S1). Perturbation of B-cell homeostasis, in particular a maturation arrest at the pro- $/$ pre-B stage, is a hallmark of human B-ALL [9]. Thus, our mouse model and the human disease show significant similarities, both in terms of differentially expressed genes and the stage of B-cell arrest. Interestingly we did not find that $\mathrm{Pax} 5$ heterozygosity accelerated leukemia development (Fig. 1a), suggesting its sole contribution to B-ALL development in our model is at the level of the induction of maturation arrest. This is in contrast to an additional cross we performed in which Etv6 $+/ R U N X 1-S B$ mice were bred to an Ink4a-deficient background resulting in a significantly decreased latency to leukemogenesis ( $\mathrm{p}=0.0012$ using a Log-rank test; Additional file 3: Figure S2), which is in agreement with reports that INK4A inactivation is associated with an aggressive clinical course in ETV6-RUNX1+ B-ALL [10].

To define common transposon insertion sites (CISs), loci in the genome that have increased clustering of transposon insertion events and hence may contain candidate driver genes, tumor DNAs from the 20 mice that developed B220+ CD19+ B-ALL with a tumour cell fraction $>60 \%$ were analysed using 454-based ligandmediated PCR sequencing [7]. Six statistically significant CISs were identified: Jak1, Stat5b, Zfp423, Il2rb, Cblb and Foxp1 (Fig. 2a). Four of these 6 genes (Zfp 423 , $C b l b$, Stat $5 b$ and Foxp 1) have well-characterised roles in regulating B-cell maturation. Analysis of the RNAseq data generated from the tumor collection confirmed that insertions in Zfp423, Jak1 and Stat5b resulted in significantly increased expression of these genes (Fig. 2a). Increased ZNF423 expression has been reported in BCP-ALL (revealing a strong association with ETV6-RUNX1+ cases) and elevated expression of this gene has been linked to a B-cell differentiation block [11]. Activation of the JAK/STAT signaling pathway is a frequent theme in hematological malignancies. In fact, increased expression of activated STAT5 is correlated with poor prognosis in ALL patients, and haploinsufficiency of Pax5 or Ebf1 synergize with constitutively expressed STAT5 to induce B-ALL [12]. Somatic mutations of $C B L / C B L B$ in B-ALL typically involve 

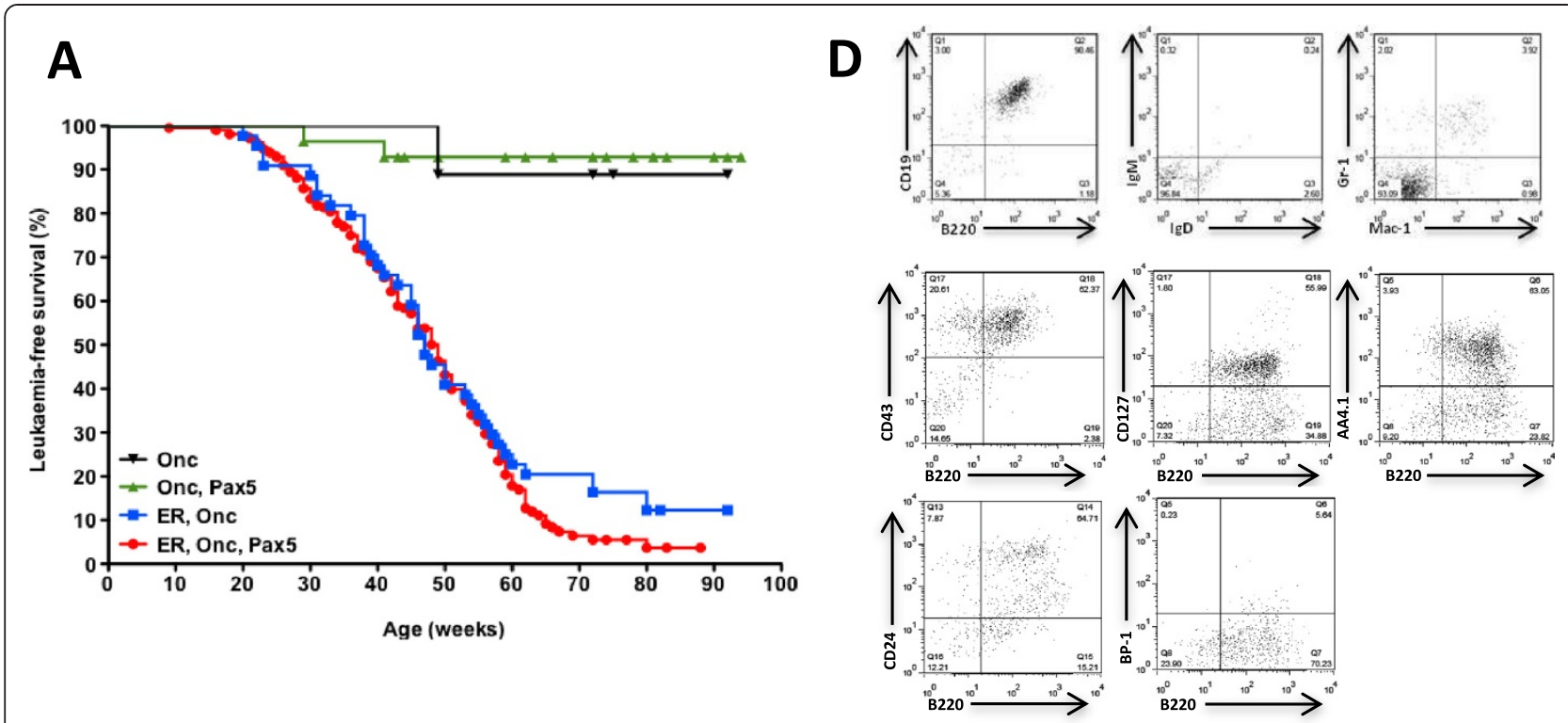

B
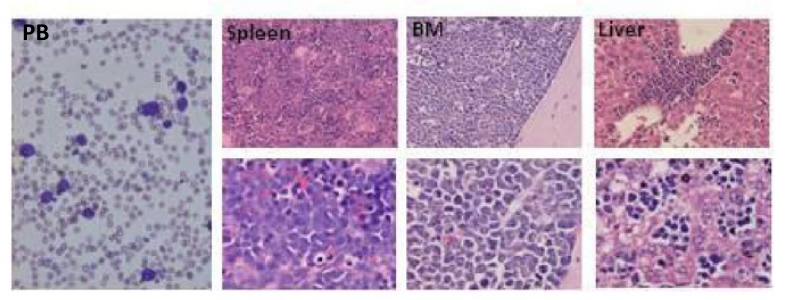

$\mathbf{E}$

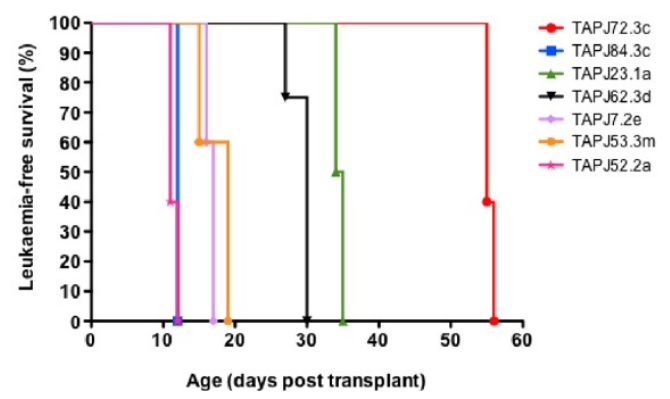

C

\begin{tabular}{l}
\hline Acute B-cell leukemia (B220+) \\
$\cdot$ B220+ cells Mac-1+ $(10-70 \%)$ \\
$\cdot$ B220+ cells CD19- \\
$\cdot$ B220+ cells CD19+ $(10-50 \%)$ \\
$\cdot$ B220+ cells CD19+ $(60-100 \%)$
\end{tabular}

Acute myeloid leukemia (Mac-1+)

Acute T-cell leukemia (CD4+)

Acute leukemia (unclassified)*

\begin{tabular}{llcc} 
ER, Onc, Pax & ER, Onc & Onc, Pax & Onc \\
\hline $60(38 \%)$ & $11(30 \%)$ & 0 & 0 \\
19 & 10 & & \\
14 & 1 & & \\
7 & 0 & & \\
20 & 0 & 0 & 1 \\
$46(29 \%)$ & $14(39 \%)$ & 0 & 0 \\
$24(15 \%)$ & $4(10 \%)$ & 2 & 0 \\
$29(18 \%)$ & $8(21 \%)$ & 2 &
\end{tabular}

*Majority of cells in population were Mac-1-, CD4-, B220-

Fig. 1 Phenotype of Etv6-RUNX1, T2Onc, Pax5 leukemias. a Kaplan-Meier curves showing the tumor latency of 'jumping' Etv6 ${ }^{+/ R U N X 1}$, T2Onc ${ }^{+\pi g}, \operatorname{Pax}^{+/-}$(ER, Onc, Pax) and Etv6 ${ }^{+R U N X 1}, T_{2 O n C^{+/ T g}}(E R$, Onc) mice, and 'non-jumping' control mice (Onc, Pax and Onc). b The presence of lymphoblasts in the peripheral blood (PB), spleen, bone marrow and liver from a representative mouse with leukemia. Magnification: peripheral blood smear $(\times 400)$, upper row organs $(\times 400)$ and lower row organs $(\times 1,000)$. c Classification of the leukemias developed by mice shown in the Kaplan-Meier curve according to the Bethesda criteria for lymphoid and non-lymphoid murine malignancies. $\mathbf{d}$ Upper row: FACS plots from the bone marrow of a representative mouse demonstrate only background Gr-1/Mac-1 myeloid cells, with the majority of cells having a B220+/CD19+/surface Ig-. Middle and lower row: FACS plots from the bone marrow of a representative mouse demonstrate the B220+ cells to have a CD43+, CD127+, AA4.1+, CD24+, BP-1- phenotype. e Survival curves for SCID mice transplanted with 3.5-5 × 105 B220+, CD19+ leukemia cells from ER, Onc, Pax mice and TAPJ23.1a which was an ER, Pax5 mouse. Each color represents a different 'primary' leukemia, as indicated by the "TAPJ" name of the mouse $(n=7)$ 
A

\begin{tabular}{cccccc}
\hline $\begin{array}{c}\text { Candidate } \\
\text { Gene }\end{array}$ & $\begin{array}{c}\text { No. tumors } \\
\text { in CIS }\end{array}$ & $\begin{array}{c}\text { No. unique } \\
\text { insertions in CIS }\end{array}$ & $\begin{array}{c}\text { RNA-seq } \\
\text { LogFC }\end{array}$ & $\begin{array}{c}\text { RNA-seq } \\
\text { P value }\end{array}$ & $\begin{array}{c}\text { RNA-seq } \\
\text { FDR }\end{array}$ \\
\hline Jak1 & 10 & 9 & +1.84 & $2.99 \times 10^{-5}$ & $2.94 \times 10^{-4}$ \\
Stat5b & 8 & 8 & +1.44 & $3.55 \times 10^{-6}$ & $2.03 \times 10^{-4}$ \\
Zfp423 & 5 & 3 & +5.43 & $1.01 \times 10^{-6}$ & $3.52 \times 10^{-1}$ \\
II2rb & 4 & 3 & -1.40 & $3.10 \times 10^{-4}$ & $4.13 \times 10^{-3}$ \\
Cblb & 5 & 4 & +0.69 & 0.034 & 0.095 \\
Foxp1 & 7 & 5 & +0.36 & 0.272 & 0.409 \\
\hline
\end{tabular}

B

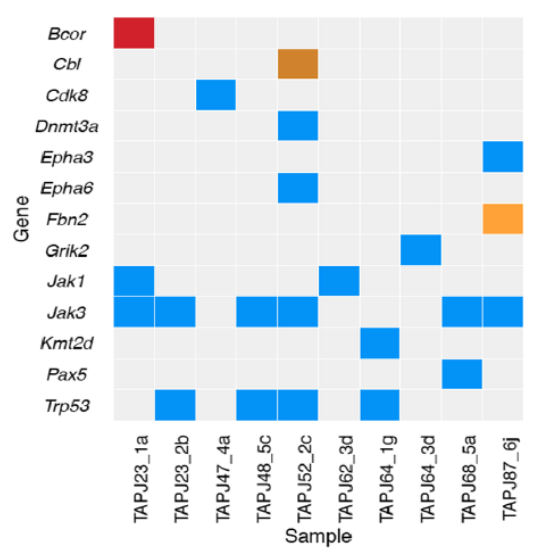

C

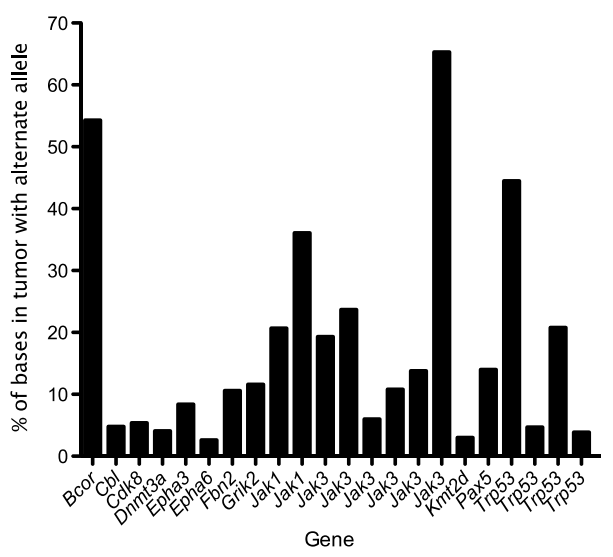

D

Jak1

\begin{tabular}{|c|c|c|c|c|}
\hline FERM & $\mathrm{SH} 2$ & Pseudokinase domain & Kinase domain & 1,153 AA \\
\hline & & 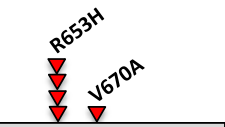 & 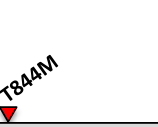 & \\
\hline FERM & SH2 & Pseudokinase domain & Kinase domain & $1,100 \mathrm{AA}$ \\
\hline
\end{tabular}

$\mathbf{E}$

\begin{tabular}{|c|c|c|c|c|}
\hline $\begin{array}{l}\text { Mouse } \\
\text { gene }\end{array}$ & $\begin{array}{l}\text { Mouse AA } \\
\text { substitution }\end{array}$ & $\begin{array}{l}\text { Corresponding } \\
\text { human AA } \\
\text { substitution }\end{array}$ & $\begin{array}{l}\text { Occurrence in } \\
\text { leukemia patients }\end{array}$ & Reference \\
\hline \multirow[t]{4}{*}{ Jak1 } & L652F & L653F & T-ALL & 17 \\
\hline & & & T-PLL & 21 \\
\hline & V657F & V658F* & B-ALL ${ }^{\star \star}$ & 18,22 \\
\hline & & & T-ALL \& AML ${ }^{* \star \star *}$ & 23 \\
\hline \multirow[t]{4}{*}{ Jak3 } & $\mathrm{R} 653 \mathrm{H}$ & $\mathrm{R}^{\prime} 57 \mathrm{Q}^{*}$ & T-ALL & 24 \\
\hline & & $\mathrm{R}^{257 Q^{*}}$ & T-PLL & 25 \\
\hline & & $\mathrm{R} 557 \mathrm{Q}^{*}$ & $\mathrm{AMKL}^{* * *}$ & 14 \\
\hline & $\begin{array}{l}\text { V670A } \\
\end{array}$ & V674F* & T-PLL & 25 \\
\hline
\end{tabular}

Fig. 2 Common insertion site and somatic mutation analysis of BCP-ALL cases in Etv6-RUNX1, T2OnC, Pax5 mice. a Transposon common insertion sites (CIS) in B-ALL cases. All CIS shown have a genome-wide $p$ value of $<0.025$. Alterations in expression levels were determined using RNA-seq data (from 20 ER, Onc, Pax mice that developed B220+, CD19+ BCP-ALL and 8 age-matched 'control' ER, Onc, Pax mice that never developed disease), whereby the expression level of a gene in BCP-ALL cases containing insertions in a particular CIS was compared to the expression level of that gene in 'control' cases. FC, log fold change; FDR, false discovery rate (significance was assessed at an FDR of 5 \%). b Acquired somatic events in B-ALL cases in ER, Onc, Pax mice. Each column represents a sample (individual mouse). Red indicates a stop mutation, brown indicates a splice acceptor variant, yellow indicates a splice region variant (intron) and blue indicates a missense variant. $\mathbf{c}$ The fraction of reads (expressed as a percentage) reporting the somatic variant alleles shown in $\mathbf{b}$ (in the same order as shown in $\mathbf{b}$ ). $\mathbf{d}$ Schematic diagram of the protein structure of Jak1 and Jak3, showing the locations of the mutations identified in this study. FERM, band 4.1 ezrin; SH2, src-homology domain. e Somatically acquired mutations in Jak1/3 found in ER, Onc, Pax B-ALL tumors in which the corresponding JAK1/3 mutations have been found in human cancers. AA, amino acid. "These mutations have been shown to have gain-of-function and/or transforming activity. ${ }^{* * *}$ These cases were pediatric high-risk B-cell progenitor ALL excluding BCR-ABL1+ ALL and hypodiploid ALL. ${ }^{* * *}$ Acute megakaryoblastic leukemia of Down syndrome. ${ }^{* * * *}$ An AML with t(15;17)(q22;q12) 
small deletions affecting the intron/exon boundaries of exon 8, leading to skipping of this exon and the abolition of E3 ligase function; the transposon insertions in Cblb were in intron 8 and thus are likely to function via a similar mechanism [13]. Mutation of CBL is an alternative route to activate the RAS pathway [13], and mutations in $R A S$ have been reported in hyperdiploidy BCP-ALL and ETV6-RUNX1+ cases [14]. Thus, our Etv6-RUNX1; $\mathrm{Pax}^{+/-}$mouse model has several cardinal genetic features associated with B-ALL.

Interestingly an Etv6+/RUNX1-SB, T2Onc ${ }^{+/+}, \mathrm{Pax}^{+/-}$ mouse (TAPJ23.1a), in which transposition was not occuring, developed transplantable B-ALL (Fig. 1e), suggesting a contribution of background somatic mutations to tumor development leading us to investigate the somatic mutation landscape by targeted exome sequencing of 404 established cancer genes and candidate BALL drivers in 17 B220 + CD19+ B-ALL cases (Fig. 2b; Additional file 1). Strikingly the most commonly mutated genes were Jak3 (6/17 mice, $35 \%)$, Trp53 (4/17 mice, $23 \%)$ and Jak1 (2/17 mice, $11 \%)$ with the missense mutations in Jak1/3 predominantly located in the pseudokinase domain (Fig. 2d). This domain has been demonstrated to exert an important negative regulatory function on the kinase domain [15] with many of the amino acid changes we identified falling into positions of JAK1/3 reported as being mutated in human leukemias, and shown to confer gain-of-function or transforming activity (Fig. 2e) [16-18]. Somatic mutations in JAK1 and JAK3 and have been reported in adult B-ALL [19] and high-risk/poor prognosis pediatric B-ALL [20], respectively. The variant allele frequency of Jak1/3 mutations was around $25 \%$ suggesting that cells with these mutations represent a major clonal fraction (Fig. 2c). Recurrent somatic mutations in Jak1/3 have recently been reported in B-ALL tumors from $\mathrm{Pax}^{+/-}$mice [PMID: 25855603], suggesting that the synergy with Jak mutations in our model is a result of the knockout allele for $\operatorname{Pax} 5$ rather than the presence of the Etv6-RUNX1 allele. We also observed somatic Trp53 mutations in our mouse tumors with copy number and/or sequence alterations of p53 being an independent risk predictor of inferior outcome/high risk of treatment failure in B-ALL patients [21, 22].

\section{Conclusions}

Collectively, our findings support a model in which multiple small defects in a network of factors that regulate B-cell maturation (such as Pax5, Cblb, Zfp423, Foxp1, and Stat5b) together with activation/inactivation of oncogenes/tumor suppressor genes (such as the JAK/STAT signaling pathway and p53) cooperate with Etv6-RUNX1; $\mathrm{Pax}^{+/-}$to result in the development BALL. Our transplantable B-ALL tumors represent a novel tool for assessing potential therapeutic intervention strategies in cases of high risk/poor outcome BALL.

\section{Additional files}

Additional file 1: List of targeted exome baits. The file contains a list of genes that were captured as part of the targeted exome sequencing performed as part of this study. (XLSX $53 \mathrm{~kb}$ )

Additional file 2: Figure S1. Pathway analysis showing enrichment for genes involved in early B-cell function in our model. (PPT 166 kb)

Additional file 3: Figure S2. Accelerated tumor development in Ink4a-1- ; Etv6 ${ }^{+/ R U N X 1-S B}$ mice. (PPT $162 \mathrm{~kb}$ )

\section{Competing interests}

The authors declare that they have no competing interests.

\section{Authors' contributions}

LvdW, GG, BJHP and DJA designed research; LvdW, GG, HO, CDR-E and HK performed research; LvdW, GG, KW, AGR, BJHP and DJA analysed data; LvdW and DJA wrote the paper; and all authors critically reviewed and edited the paper. All authors read and approved the final manuscript.

\section{Acknowledgements}

The authors wish to thank the staff of the Research Support Facility at the Wellcome Trust Sanger Institute for looking after the mice.

L.v.d.W., K.W., A.G.R., C.D.R.-E., and D.J.A. were supported by Cancer Research UK and the Wellcome Trust (WT098051). C.D.R.-E. was also supported by the Consejo Nacional de Ciencia y Tecnología of Mexico. B.J.H. and G.G. were supported by Medical Research Council UK and Cancer Research UK.

\section{Author details}

${ }^{1}$ Wellcome Trust Sanger Institute, Wellcome Trust Genome Campus, Hinxton, Cambridge CB10 1HH, UK. ${ }^{2}$ Cambridge Institute for Medical Research and Wellcome Trust/MRC Cambridge Stem Cell Institute, Wellcome Trust/MRC Building, Addenbrooke's Hospital, Hills Road, Cambridge CB2 OXY, UK. ${ }^{3}$ Experimental Cancer Genetics, Wellcome Trust Sanger Institute, Wellcome Trust Genome Campus, Hinxton, Cambridge CB10 1HH, UK.

Received: 26 April 2015 Accepted: 27 July 2015

Published online: 13 August 2015

\section{References}

1. Mullighan CG. Molecular genetics of B-precursor acute lymphoblastic leukemia. J Clin Invest. 2012;122:3407-15.

2. Shurtleff SA, Buijs A, Behm FG, Rubnitz JE, Raimondi SC, Hancock ML, et al. TEL/AML1 fusion resulting from a cryptic $t(12 ; 21)$ is the most common genetic lesion in pediatric ALL and defines a subgroup of patients with an excellent prognosis. Leukemia. 1995;9:1985-9.

3. Ford AM, Bennett CA, Price CM, Bruin MC, Van Wering ER, Greaves M. Fetal origins of the TEL-AML1 fusion gene in identical twins with leukemia. Proc Natl Acad Sci U S A. 1998:95:4584-8.

4. Greaves MF, Maia AT, Wiemels JL, Ford AM. Leukemia in twins: lessons in natural history. Blood. 2003;102:2321-33.

5. Mullighan CG, Goorha S, Radtke I, Miller CB, Coustan-Smith E, Dalton JD, et al. Genome-wide analysis of genetic alterations in acute lymphoblastic leukaemia. Nature. 2007;446:758-64.

6. Lilljebjörn H, Soneson C, Andersson A, Heldrup J, Behrendtz M, Kawamata N, et al. The correlation pattern of acquired copy number changes in 164 ETV6/RUNX1-positive childhood acute lymphoblastic leukemias. Hum Mol Genet. 2010;19:3150-8.

7. Van der Weyden L, Giotopoulos G, Rust AG, Matheson LS, van Delft FW, Kong J, et al. Modeling the evolution of ETV6-RUNX1-induced B-cell precursor acute lymphoblastic leukemia in mice. Blood. 2011;118:1041-51.

8. Urbánek P, Wang ZQ, Fetka I, Wagner EF, Busslinger M. Complete block of early $B$ cell differentiation and altered patterning of the posterior midbrain in mice lacking Pax5/BSAP. Cell. 1994;79:901-12. 
9. Zhou Y, You MJ, Young KH, Lin P, Lu G, Medeiros LJ, et al. Advances in the molecular pathobiology of B-lymphoblastic leukemia. Hum Pathol. 2012;43:1347-62.

10. Papadhimitriou SI, Polychronopoulou S, Tsakiridou AA, Androutsos G, Paterakis GS, Athanassiadou F. p16 inactivation associated with aggressive clinical course and fatal outcome in TEL/AML1-positive acute lymphoblastic leukemia. J Pediatr Hematol Oncol. 2005;27:675-7.

11. Harder L, Eschenburg G, Zech A, Kriebitzsch N, Otto B, Streichert T, et al. Aberrant ZNF423 impedes B cell differentiation and is linked to adverse outcome of ETV6-RUNX1 negative B precursor acute lymphoblastic leukemia. J Exp Med. 2013;210:2289-304.

12. Heltemes-Harris LM, Willette MJL, Ramsey LB, Qiu YH, Neeley ES, Zhang N, et al. Ebf1 or Pax5 haploinsufficiency synergizes with STAT5 activation to initiate acute lymphoblastic leukemia. J Exp Med. 2011;208:1135-49.

13. Nicholson L, Knight T, Matheson E, Minto L, Case M, Sanichar M, et al. Casitas B lymphoma mutations in childhood acute lymphoblastic leukemia. Gene Chromosome Canc. 2012;51:250-6.

14. Case M, Matheson E, Minto L, Hassan R, Harrison CJ, Bown N, et al. Mutation of genes affecting the RAS pathway is common in childhood acute lymphoblastic leukemia. Cancer Res. 2008;68:6803-9.

15. Saharinen $P$, Vihinen $M$, Silvennoinen O. Autoinhibition of Jak2 tyrosine kinase is dependent on specific regions in its pseudokinase domain. Mol Biol Cell. 2003;14:1448-59.

16. Sato T, Toki T, Kanezaki R, Xu G, Terui K, Kanegane H, et al. Functional analysis of JAK3 mutations in transient myeloproliferative disorder and acute megakaryoblastic leukaemia accompanying Down syndrome. Br J Haematol. 2008;141:681-8.

17. Choi YL, Kaneda R, Wada T, Fujiwara S-I, Soda M, Watanabe H, et al. Identification of a constitutively active mutant of JAK3 by retroviral expression screening. Leuk Res. 2007;31:203-9.

18. Staerk J, Kallin A, Demoulin J-B, Vainchenker W, Constantinescu SN. JAK1 and Tyk2 activation by the homologous polycythemia vera JAK2 V617F mutation: cross-talk with IGF1 receptor. J Biol Chem. 2005;280:41893-9.

19. Flex E, Petrangeli V, Stella L, Chiaretti S, Hornakova T, Knoops L, et al. Somatically acquired JAK1 mutations in adult acute lymphoblastic leukemia. J Exp Med. 2008;205:751-8.

20. Mullighan CG, Zhang J, Harvey RC, Collins-Underwood JR, Schulman BA Phillips LA, et al. JAK mutations in high-risk childhood acute lymphoblastic leukemia. Proc Natl Acad Sci U S A. 2009;106:9414-8.

21. Hof J, Krentz S, van Schewick C, Körner G, Shalapour S, Rhein P, et al. Mutations and deletions of the TP53 gene predict nonresponse to treatment and poor outcome in first relapse of childhood acute lymphoblastic leukemia. J Clin Oncol Off J Am Soc Clin Oncol. 2011;29:3185-93.

22. Krentz S, Hof J, Mendioroz A, Vaggopoulou R, Dörge P, Lottaz C, et al. Prognostic value of genetic alterations in children with first bone marrow relapse of childhood B-cell precursor acute lymphoblastic leukemia. Leukemia. 2013;27:295-304.

\section{Submit your next manuscript to BioMed Central and take full advantage of:}

- Convenient online submission

- Thorough peer review

- No space constraints or color figure charges

- Immediate publication on acceptance

- Inclusion in PubMed, CAS, Scopus and Google Scholar

- Research which is freely available for redistribution 